ALEA, Lat. Am. J. Probab. Math. Stat. 18, 193-209 (2021)

DOI: 10.30757/ALEA.v18-09

\title{
A stochastic Gronwall lemma and well-posedness of path-dependent SDEs driven by martingale noise
}

\author{
Sima Mehri and Michael Scheutzow
}

\author{
Institut für Mathematik \\ Humboldt Universität zu Berlin \\ Unter den Linden 6 \\ D-10099 Berlin, Germany. \\ E-mail address: sima.mehri@math.hu-berlin.de \\ $U R L$ : https://www.applied-financial-mathematics.de/sima-mehri \\ Institut für Mathematik \\ Technische Universität Berlin \\ Straße des 17. Juni 136 \\ D-10623 Berlin, Germany. \\ E-mail address: ms@math.tu-berlin.de \\ URL: http://page.math.tu-berlin.de/ scheutzow/
}

\begin{abstract}
We show existence and uniqueness of solutions of stochastic path dependent differential equations driven by càdlàg martingale noise under joint local monotonicity and coercivity assumptions on the coefficients with a bound in terms of the supremum norm. In this set-up the usual proof using the ordinary Gronwall lemma together with the Burkholder-Davis-Gundy inequality seems impossible. In order to solve this problem, we prove a new and quite general stochastic Gronwall lemma for càdlàg martingales using Lenglart's inequality.
\end{abstract}

Received by the editors August 28th, 2019; accepted October 28th, 2020.

2010 Mathematics Subject Classification. 34K50, 60H10, 60G57, 65L03, 60G44.

Key words and phrases. Stochastic Gronwall Lemma, Functional Stochastic Differential Equations, Path-Dependent Stochastic Differential Equations, Martingale Inequality, Monotone Coefficients, Lenglart Inequality.

The work of first author was supported by the Hilda Geiringer Scholarship awarded by the Berlin Mathematical School. 


\section{Introduction}

Fix $\tau>0$ and let $\left(\Omega, \mathcal{F},\left(\mathcal{F}_{t}\right)_{t \geq 0}, \mathbb{P}\right)$ be a normal filtered probability space, i.e. the space is complete and satisfies the usual conditions. Consider the following stochastic delay differential equation in $\mathbb{R}^{d}$ :

$$
\left\{\begin{array}{l}
\mathrm{d} X(t)=f\left(t, \omega, X_{t-\tau: t}\right) \mathrm{d} t+\int_{U} g\left(t, \omega, X_{t-\tau: t}, \xi\right) \tilde{M}(\mathrm{~d} t, \mathrm{~d} \xi), \\
X(t)=z(t), \quad t \in[-\tau, 0]
\end{array}\right.
$$

where $X_{t-\tau: t}(s)=X(t+s), s \in[-\tau, 0]$ and $z \in L^{2}\left(\Omega, \mathcal{F}_{0}, \mathbb{P} ; \operatorname{Càdlàg}\left([-\tau, 0], \mathbb{R}^{d}\right)\right)$.

We will state precise assumptions on the coefficients $f$ and $g$ and on the martingale measure $\tilde{M}$ in Section 3. For the moment, assume that $U=U_{1} \sqcup U_{2}$, where $U_{1}$ is a finite or infinite subset of $\mathbb{N}$ and the integral over $U_{1}$ is a sum, where $\tilde{M}_{t}(i)$, $i \in U_{1}$ are independent Wiener processes and the remaining integral over $U_{2}$ is with respect to compensated Poisson noise which is independent of the Wiener processes. If $U_{2}=\emptyset$, then we speak of Wiener or diffusive noise, otherwise of jump diffusive noise. In the diffusive case, several authors established existence and uniqueness of solutions of (1.1) under various conditions on the coefficients (e.g. Mao, 2008, Theorem 5.2.5 under local Lipschitz and linear growth assumptions on $f$ and $g$ and von Renesse and Scheutzow, 2010 under a one-sided local Lipschitz and a suitable growth condition). Under similar conditions, Fengying and Ke (2007) and Ren et al. (2008) show existence and uniqueness even for equations with infinite delay and Mohammed and Scheutzow (2003) (see also Chueshov and Scheutzow, 2013) proved not only existence and uniqueness but also pathwise continuous dependence of the solution on the initial condition in case $g$ does not depend on the past (otherwise it is known that pathwise continuous dependence on the initial condition does not hold in general, see Mohammed and Scheutzow, 1997). Existence and uniqueness results in the jump diffusive case under a local Lipschitz and linear growth condition (even with additional Markovian switching) were obtained in Zhu (2017).

In both the existence and the uniqueness proof one typically encounters the following inequality for some non-negative adapted process $Z$,

$$
Z(t) \leq K \int_{0}^{t} Z^{\star}(s) \mathrm{d} s+M(t)+H(t),
$$

where $Z^{\star}(s)=\sup _{u \in[0, s]} Z(u), M$ is a local martingale (depending on the function $g$ in the equation), the process $H(t), t \geq 0$ is non-decreasing adapted, and $K>0$ is a constant. In order to apply Gronwall's lemma, the expression inside the integral should be the same as the expression on the left side of the inequality. Taking the supremum on both sides of (1.2) and then taking expectations, an upper bound for $\mathbb{E} M^{\star}(t)$ in terms of the process $Z$ is required. Under a local one-sided Lipschitz condition of the form

For all compact subset $\mathcal{C} \subset C\left([-\tau, 0], \mathbb{R}^{d}\right)$ there exists $L_{\mathcal{C}}>0$ and

$$
\begin{gathered}
\tau_{\mathcal{C}} \in(-\tau, 0] \text { such that } \forall x, y \in \mathcal{C} \text { with } x(s)=y(s) \forall s \in\left[-\tau,-\tau_{\mathcal{C}}\right] \\
2\langle x(0)-y(0), f(x)-f(y)\rangle+|g(x)-g(y)|^{2} \leq L_{\mathcal{C}} \sup _{s \in[-\tau, 0]}|x(s)-y(s)|^{2},
\end{gathered}
$$

as in von Renesse and Scheutzow (2010), controls with respect to the supremum norm on $g$ are not separated from $f$ and it therefore seems impossible to use the 
Burkholder-Davis-Gundy inequality to obtain an upper bound for $\mathbb{E} M^{\star}(t)$ in this case.

von Renesse and Scheutzow (2010) dealt with this problem by proving the following stochastic Gronwall's inequality for the above mentioned process $Z$ and for $p \in(0,1)$ and $\alpha>\frac{1+p}{1-p}$ :

$$
\mathbb{E}\left[\left(Z^{\star}(T)\right)^{p}\right] \leq c_{1} e^{c_{2} K T}\left(\mathbb{E}\left[H(T)^{\alpha}\right]\right)^{p / \alpha}, \quad \forall T \geq 0 .
$$

Here $c_{1}$ and $c_{2}$ are two constants that only depend on $p$ and $\alpha$ and $Z, H$, and $M$ are assumed to have continuous paths (in addition to the properties stated above).

One can find another type of stochastic Gronwall lemma in the literature where $Z^{\star}(s) K \mathrm{~d} s$ in the assumption is replaced by $Z\left(s^{-}\right) \mathrm{d} A(s)$ for an adapted nondecreasing stochastic process $A$ (see Scheutzow, 2013 for continuous processes, Zhang and Zhao, 2018 for càdlàg processes and Kruse and Scheutzow, 2018 for discrete time processes).

Whenever the supremum norm in condition (1.3) is replaced by a real-valued continuous linear operator, say $\lambda$, on $\operatorname{Càdlàg}([-\tau, 0], \mathbb{R})$, then there is no problem using the ordinary Gronwall's lemma. In Mehri et al. (2020), we have stated the well-posedness of equation (1.1) driven by jump diffusion under the local monotonicity assumption,

$$
\begin{gathered}
\forall R>0, \exists L_{R} \in L_{l o c}^{1}\left(\mathbb{R}_{\geq 0}, \mathbb{R}_{\geq 0}\right), \forall x, y \in \text { Càdlàg }\left([-\tau, 0], \mathbb{R}^{d}\right) \\
\text { with } \sup _{s \in[-\tau, 0]}|x(s)|, \sup _{s \in[-\tau, 0]}|y(s)|<R: \\
2\left\langle x\left(0^{-}\right)-y\left(0^{-}\right), f(t, \omega, x)-f(t, \omega, y)\right\rangle+\int_{U}|g(t, \omega, x, \xi)-g(t, \omega, y, \xi)|^{2} \nu_{t}(\mathrm{~d} \xi) \\
\leq L_{R}(t) \lambda\left(|x(\cdot)-y(\cdot)|^{2}\right)
\end{gathered}
$$

and coercivity assumption,

$$
\begin{gathered}
\exists K \in L_{l o c}^{1}\left(\mathbb{R}_{\geq 0}, \mathbb{R}_{\geq 0}\right), \forall x \in \operatorname{Càdlàg}\left([-\tau, 0], \mathbb{R}^{d}\right): \\
2\left\langle x\left(0^{-}\right), f(t, \omega, x)\right\rangle+\int_{U}|g(t, \omega, x, \xi)|^{2} \nu_{t}(\mathrm{~d} \xi) \leq K(t) \lambda\left(1+|x(\cdot)|^{2}\right)
\end{gathered}
$$

without using a stochastic Gronwall lemma. We will provide a simple example of an equation satisfying our assumptions but not those in previous work in Section 4.

In this paper, we study existence and uniqueness of equation

$$
\left\{\begin{array}{l}
\mathrm{d} X(t)=f(t, \omega, X) \mathrm{d} t+\int_{U} g(t, \omega, X, \xi) \tilde{M}(\mathrm{~d} t, \mathrm{~d} \xi), \\
X(t)=z(t), \quad t \in[-\tau, 0]
\end{array}\right.
$$

under weaker conditions than those stated above. In particular, $\tilde{M}$ will be a rather general martingale measure, and $f$ and $g$ satisfy weaker conditions than (1.4) and (1.5), namely the right hand sides are replaced by the supremum norm. We will state precise conditions later.

\section{Stochastic Gronwall Lemma}

Throughout this section, we will assume that $(\Omega, \mathcal{F}, \mathbb{P})$ is a probability space with normal filtration $\left(\mathcal{F}_{t}\right)_{t \geq 0}$. We will prove the following theorem. 
Theorem 2.1 (Stochastic Gronwall lemma). Let $Z(t), t \geq 0$ be an $\left(\mathcal{F}_{t}\right)_{t>0}$-adapted non-negative right-continuous process. Assume that $A:[0, \infty) \rightarrow[0, \infty)$ is a deterministic non-decreasing càdlàg function with $A(0)=0$ and let $H(t), t \geq 0$ be a non-decreasing and càdlàg adapted process starting from $H(0) \geq 0$. Further, let $M(t), t \geq 0$ be an $\left(\mathcal{F}_{t}\right)_{t \geq 0}$ - local martingale with $M(0)=0$ and càdlàg paths. Assume that for all $t \geq 0$,

$$
Z(t) \leq \int_{0}^{t} Z^{*}\left(u^{-}\right) \mathrm{d} A(u)+M(t)+H(t)
$$

where $Z^{*}(u):=\sup _{r \in[0, u]} Z(r)$. Then the following estimates hold for $p \in(0,1)$ and $T>0$.

(a) If $\mathbb{E}\left(H(T)^{p}\right)<\infty$ and $H$ is predictable, then

$$
\mathbb{E}\left[\left(Z^{*}(T)\right)^{p} \mid \mathcal{F}_{0}\right] \leq \frac{c_{p}}{p} \mathbb{E}\left[(H(T))^{p} \mid \mathcal{F}_{0}\right] \exp \left\{c_{p}^{1 / p} A(T)\right\} .
$$

(b) If $\mathbb{E}\left(H(T)^{p}\right)<\infty$ and $M$ has no negative jumps, then

$$
\mathbb{E}\left[\left(Z^{*}(T)\right)^{p} \mid \mathcal{F}_{0}\right] \leq \frac{c_{p}+1}{p} \mathbb{E}\left[(H(T))^{p} \mid \mathcal{F}_{0}\right] \exp \left\{\left(c_{p}+1\right)^{1 / p} A(T)\right\} .
$$

(c) If $\mathbb{E} H(T)<\infty$, then

$$
\mathbb{E}\left[\left(Z^{*}(T)\right)^{p} \mid \mathcal{F}_{0}\right] \leq \frac{c_{p}}{p}\left(\mathbb{E}\left[H(T) \mid \mathcal{F}_{0}\right]\right)^{p} \exp \left\{c_{p}^{1 / p} A(T)\right\} .
$$

Here $c_{p}=\frac{p^{-p}}{1-p}$.

For the proof of Theorem 2.1, we will need the following lemma which is essentially Lenglart (1977, Théorème I \& Corollaire II) with a slightly better constant $c_{p}$ and slightly weaker assumptions. Note that Revuz and Yor (1999, Proposition IV.4.7 \& Exercise IV.4.30) states a similar result for the case of continuous $G$ and Ren and Shen (2012) for general $G$.

Lemma 2.2. Let $Z$ be a non-negative adapted right-continuous process and let $G$ be a non-negative right-continuous non-decreasing predictable process such that $\mathbb{E}\left[Z(\tau) \mid \mathcal{F}_{0}\right] \leq \mathbb{E}\left[G(\tau) \mid \mathcal{F}_{0}\right] \leq \infty$ for any bounded stopping time $\tau$. Then

(i) $\forall c, d>0$,

$$
\mathbb{P}\left(\sup _{t \geq 0} Z(t)>c \mid \mathcal{F}_{0}\right) \leq \frac{1}{c} \mathbb{E}\left[\sup _{t \geq 0} G(t) \wedge d \mid \mathcal{F}_{0}\right]+\mathbb{P}\left(\sup _{t \geq 0} G(t) \geq d \mid \mathcal{F}_{0}\right) .
$$

(ii) For all $p \in(0,1)$,

$$
\mathbb{E}\left[\left(\sup _{t \geq 0} Z(t)\right)^{p} \mid \mathcal{F}_{0}\right] \leq c_{p} \mathbb{E}\left[\left(\sup _{t \geq 0} G(t)\right)^{p} \mid \mathcal{F}_{0}\right],
$$

where $c_{p}:=\frac{p^{-p}}{1-p}$.

For the proof of this lemma, recall that a predictable stopping time is a map $\tau: \Omega \rightarrow[0, \infty]$ for which there exists an increasing sequence $\left(\tau_{n}\right)_{n \in \mathbb{N}}$ of stopping times (called announcing sequence for $\tau$ ) with the properties

(a) $\lim _{n \rightarrow \infty} \tau_{n}(\omega)=\tau(\omega), \forall \omega \in \Omega$,

(b) $\tau_{n}(\omega)<\tau(\omega), \forall \omega \in\{\tau>0\}$ 
(see Dellacherie, 1972, p56). For $B \subset[0, \infty) \times \Omega$, let $T_{B}(\omega):=\inf \{t \geq 0:(t, \omega) \in B\}$ be the first hitting time of $B$. If $B$ is predictable and $\left\{(t, \omega): T_{B}(\omega)=t\right\} \subset B$, then $T_{B}$ is a predictable stopping time (Dellacherie, 1972, p74).

Proof of Lemma 2.2, Part (i): This is essentially Theorem I in Lenglart (1977) with two small modifications: both the assumption and the conclusion in Lenglart (1977) are formulated for expected values rather than conditional expectations and Lenglart (1977) assumes that $G(0)=0$ almost surely which we do not assume. Both generalizations are easy to see but for the convenience of the reader we provide a proof.

Let $\tilde{\tau}_{d}:=\inf \{t \geq 0: G(t) \geq d\}$ and $\tau_{c}:=\inf \{t \geq 0: Z(t) \geq c\}$. Since $G$ is a predictable process, $\tilde{\tau}_{d}$ is the first hitting time of the predictable set $B=\{(t, \omega)$ : $G(t, \omega) \geq d\}$ and hence is a predictable stopping time since $\left\{(t, \omega): \tilde{\tau}_{d}(\omega)=t\right\} \subset B$. Therefore, there exists a sequence of stopping times $\tilde{\tau}_{d}^{n}, n \in \mathbb{N}$ such that $\tilde{\tau}_{d}^{n} \uparrow \tilde{\tau}_{d}$ as $n \uparrow \infty$ and $\tilde{\tau}_{d}^{n}<\tilde{\tau}_{d}$ for all $n \in \mathbb{N}$ on $\left\{\tilde{\tau}_{d}>0\right\}=\{G(0)<d\}$. Then for $T>0$,

$$
\begin{aligned}
& \mathbb{P}\left(\sup _{t \in[0, T]} Z(t)>c \mid \mathcal{F}_{0}\right) \\
& \quad=\mathbb{P}\left(\sup _{t \in[0, T]} Z(t)>c, G(T)<d \mid \mathcal{F}_{0}\right)+\mathbb{P}\left(\sup _{t \in[0, T]} Z(t)>c, G(T) \geq d \mid \mathcal{F}_{0}\right) \\
& \quad \leq \mathbb{P}\left(\left\{\mathbf{1}_{\{G(0)<d\}} Z\left(T \wedge \tau_{c}\right) \geq c\right\} \cap\left\{\tilde{\tau}_{d}>T\right\} \mid \mathcal{F}_{0}\right)+\mathbb{P}\left(G(T) \geq d \mid \mathcal{F}_{0}\right) \\
& \quad=\lim _{n \rightarrow \infty} \mathbb{P}\left(\left\{\mathbf{1}_{\{G(0)<d\}} Z\left(T \wedge \tau_{c}\right) \geq c\right\} \cap\left\{\tilde{\tau}_{d}^{n}>T\right\} \mid \mathcal{F}_{0}\right)+\mathbb{P}\left(G(T) \geq d \mid \mathcal{F}_{0}\right) \\
& \quad=\lim _{n \rightarrow \infty} \mathbb{P}\left(\left\{\mathbf{1}_{\{G(0)<d\}} Z\left(T \wedge \tilde{\tau}_{d}^{n} \wedge \tau_{c}\right) \geq c\right\} \cap\left\{\tilde{\tau}_{d}^{n}>T\right\} \mid \mathcal{F}_{0}\right)+\mathbb{P}\left(G(T) \geq d \mid \mathcal{F}_{0}\right) \\
& \quad \leq \lim _{n \rightarrow \infty} \mathbb{P}\left(\left\{\mathbf{1}_{\{G(0)<d\}} Z\left(T \wedge \tilde{\tau}_{d}^{n} \wedge \tau_{c}\right) \geq c\right\} \mid \mathcal{F}_{0}\right)+\mathbb{P}\left(G(T) \geq d \mid \mathcal{F}_{0}\right) \\
& \quad \leq \frac{1}{c} \lim _{n \rightarrow \infty} \mathbb{E}\left[\mathbf{1}_{\{G(0)<d\}} G\left(T \wedge \tilde{\tau}_{d}^{n} \wedge \tau_{c}\right) \mid \mathcal{F}_{0}\right]+\mathbb{P}\left(G(T) \geq d \mid \mathcal{F}_{0}\right) \\
& \quad \leq \frac{1}{c} \mathbb{E}\left[G(T) \wedge d \mid \mathcal{F}_{0}\right]+\mathbb{P}\left(G(T) \geq d \mid \mathcal{F}_{0}\right)
\end{aligned}
$$

Taking the limit $T \rightarrow+\infty$ the result follows.

Proof of Lemma 2.2, Part (ii): Using part (i), we have, for $\lambda>0$,

$$
\begin{aligned}
\mathbb{E}\left[\left(\sup _{t \geq 0} Z(t)\right)^{p} \mid \mathcal{F}_{0}\right]=\int_{0}^{+\infty} \mathbb{P}\left(\sup _{t \geq 0} Z(t)>c^{1 / p} \mid \mathcal{F}_{0}\right) \mathrm{d} c \\
\leq \int_{0}^{+\infty}\left\{\frac{1}{c^{1 / p}} \mathbb{E}\left[\sup _{t \geq 0} G(t) \wedge \lambda c^{1 / p} \mid \mathcal{F}_{0}\right]+\mathbb{P}\left(\sup _{t \geq 0} G(t) \geq \lambda c^{1 / p} \mid \mathcal{F}_{0}\right)\right\} \mathrm{d} c \\
=\mathbb{E}\left[\int_{0}^{\left(\sup _{t \geq 0} G(t) / \lambda\right)^{p}} \lambda \mathrm{d} c+\int_{\left(\sup _{t \geq 0} G(t) / \lambda\right)^{p}}^{+\infty} \frac{\sup _{t \geq 0} G(t)}{c^{1 / p}} \mathrm{~d} c \mid \mathcal{F}_{0}\right] \\
+\lambda^{-p} \mathbb{E}\left[\left(\sup _{t \geq 0} G(t)\right)^{p} \mid \mathcal{F}_{0}\right] \\
=\left(\frac{1}{1-p} \lambda^{1-p}+\lambda^{-p}\right) \mathbb{E}\left[\left(\sup _{t \geq 0} G(t)\right)^{p} \mid \mathcal{F}_{0}\right]
\end{aligned}
$$

The minimal value of $(1-p)^{-1} \lambda^{1-p}+\lambda^{-p}$ is equal to $c_{p}$ for the minimizer $\lambda=p$. 
Proof of Theorem 2.1: Note that the usual Gronwall lemma and (2.1) imply that $Z$ is almost surely locally bounded since this holds true for $M$ and $H$ (observe that we did not assume that $Z$ has left limits).

Part (a) Let $\sigma_{n}, n \in \mathbb{N}$ be a localizing sequence of stopping times for the local martingale $M$ and define $\tau_{n}:=\inf \{t \geq 0: X(t)>n\} \wedge \sigma_{n}$. Then it holds that

$$
\begin{aligned}
Z\left(t \wedge \tau_{n}\right) & \leq \int_{0}^{t} Z^{*}\left(\left(s \wedge \tau_{n}\right)^{-}\right) \mathrm{d} A(s)+M\left(t \wedge \tau_{n}\right)+H(t) \\
& \leq \int_{0}^{t} Z^{*}\left(s^{-} \wedge \tau_{n}\right) \mathrm{d} A(s)+M\left(t \wedge \tau_{n}\right)+H(t),
\end{aligned}
$$

$Z$ is a nonnegative right-continuous process and

$$
G_{n}(t):=\int_{0}^{t} Z^{*}\left(s^{-} \wedge \tau_{n}\right) \mathrm{d} A(s)+H(t)
$$

is non-decreasing and predictable with the property that for every finite stopping time $\tau$, we have $\mathbb{E}\left[Z\left(\tau \wedge \tau_{n}\right) \mid \mathcal{F}_{0}\right] \leq \mathbb{E}\left[G_{n}(\tau) \mid \mathcal{F}_{0}\right] \leq \infty$. Therefore, using Lemma 2.2 and Young's inequality, we have, for $\lambda>0$ and $t \geq 0$

$$
\begin{aligned}
& \mathbb{E}\left[\left(Z^{*}\left(t \wedge \tau_{n}\right)\right)^{p} \mid \mathcal{F}_{0}\right] \\
& \leq c_{p} \mathbb{E}\left[\left(\int_{0}^{t} Z^{*}\left(s^{-} \wedge \tau_{n}\right) \mathrm{d} A(s)\right)^{p}+(H(t))^{p} \mid \mathcal{F}_{0}\right] \\
& \leq c_{p} \mathbb{E}\left[\left(\int_{0}^{t}\left(Z^{*}\left(s^{-} \wedge \tau_{n}\right)\right)^{p} \mathrm{~d} A(s)\right)^{p}\left(Z^{*}\left(t^{-} \wedge \tau_{n}\right)\right)^{p(1-p)}+(H(t))^{p} \mid \mathcal{F}_{0}\right] \\
& \leq c_{p} \mathbb{E}\left[p \lambda^{1-p} \int_{0}^{t}\left(Z^{*}\left(s^{-} \wedge \tau_{n}\right)\right)^{p} \mathrm{~d} A(s)+(1-p) \lambda^{-p}\left(Z^{*}\left(t \wedge \tau_{n}\right)\right)^{p}+(H(t))^{p} \mid \mathcal{F}_{0}\right] .
\end{aligned}
$$

It follows from the first inequality in (2.5) that $\mathbb{E}\left[\left(Z^{*}\left(T \wedge \tau_{n}\right)\right)^{p} \mid \mathcal{F}_{0}\right]<\infty$ almost surely. Hence, applying the usual Gronwall's lemma to $f(t):=\mathbb{E}\left(Z^{*}\left(t \wedge \tau_{n}\right)^{p} \mid \mathcal{F}_{0}\right)$, we get for $\lambda>c_{p}^{1 / p}(1-p)^{1 / p}$,

$$
\mathbb{E}\left[\left(Z^{*}\left(T \wedge \tau_{n}\right)\right)^{p} \mid \mathcal{F}_{0}\right] \leq \exp \left(\frac{c_{p} p \lambda^{1-p} A(T)}{1-c_{p}(1-p) \lambda^{-p}}\right) \frac{c_{p} \mathbb{E}\left[(H(T))^{p} \mid \mathcal{F}_{0}\right]}{1-c_{p}(1-p) \lambda^{-p}},
$$

so applying Fatou's lemma, we get

$$
\begin{aligned}
\mathbb{E}\left[\left(Z^{*}(T)\right)^{p} \mid \mathcal{F}_{0}\right] & \leq \liminf _{n \rightarrow+\infty} \mathbb{E}\left[\left(Z^{*}\left(T \wedge \tau_{n}\right)\right)^{p} \mid \mathcal{F}_{0}\right] \\
& \leq \exp \left(\frac{c_{p} p \lambda^{1-p} A(T)}{1-c_{p}(1-p) \lambda^{-p}}\right) \frac{c_{p} \mathbb{E}\left[(H(T))^{p} \mid \mathcal{F}_{0}\right]}{1-c_{p}(1-p) \lambda^{-p}}
\end{aligned}
$$

which yields inequality (2.2) by taking $\lambda=c_{p}^{1 / p}$.

Part (b). Let $\sigma_{n}, n \in \mathbb{N}$ be a localizing sequence of stopping times for the continuous local martingale $M$ and define $\tau_{n}:=\inf \{t \geq 0: Z(t)>n\} \wedge \sigma_{n}$. Then it holds that

$$
\tilde{G}_{n}(t):=-\inf _{s \in[0, t]} M\left(s \wedge \tau_{n}\right) \leq \int_{0}^{t} Z^{*}\left(\left(s \wedge \tau_{n}\right)^{-}\right) \mathrm{d} A(s)+H(t),
$$

$M\left(t \wedge \tau_{n}\right)+\tilde{G}_{n}(t), t \geq 0$ is a nonnegative continuous process and $\tilde{G}_{n}$ is nondecreasing and predictable with the property that for every bounded stopping time 
$\tau, \mathbb{E}\left[M\left(\tau \wedge \tau_{n}\right) \mid \mathcal{F}_{0}\right] \leq \mathbb{E}\left[\tilde{G}_{n}(\tau) \mid \mathcal{F}_{0}\right]$. Therefore using Lemma 2.2, we have

$$
\mathbb{E}\left[\left(\sup _{s \in[0, t]} M\left(s \wedge \tau_{n}\right)\right)^{p} \mid \mathcal{F}_{0}\right] \leq c_{p} \mathbb{E}\left[\left(\int_{0}^{t} Z^{*}\left(\left(s \wedge \tau_{n}\right)^{-}\right) \mathrm{d} A(s)+H(t)\right)^{p} \mid \mathcal{F}_{0}\right] .
$$

Using inequality (2.5), we get

$$
\mathbb{E}\left[\left(Z^{*}\left(t \wedge \tau_{n}\right)\right)^{p} \mid \mathcal{F}_{0}\right] \leq\left(c_{p}+1\right) \mathbb{E}\left[\left(\int_{0}^{t} Z^{*}\left(\left(s \wedge \tau_{n}\right)^{-}\right) \mathrm{d} A(s)+H(t)\right)^{p} \mid \mathcal{F}_{0}\right] .
$$

The rest of the proof is similar to the proof of part (a).

Part (c). Now we prove the inequality for general $H$. Defining the new local martingale

$$
\tilde{M}(t):=M(t)+\mathbb{E}\left[H(T) \mid \mathcal{F}_{t}\right]-\mathbb{E}\left[H(T) \mid \mathcal{F}_{0}\right]
$$

(where we take a càdlàg modification of $t \mapsto \mathbb{E}\left[H(T) \mid \mathcal{F}_{t}\right]$ ) and the predictable process $\tilde{H}(t):=\mathbb{E}\left[H(T) \mid \mathcal{F}_{0}\right]$, we have

$$
Z(t) \leq \int_{0}^{t} Z^{*}\left(u^{-}\right) \mathrm{d} A(u)+\tilde{M}(t)+\tilde{H}(t),
$$

since $\mathbb{E}\left[H(T) \mid \mathcal{F}_{t}\right] \geq H(t)$. Thus the result follows from part (a).

Remark 2.3. Lemma 5.4 in von Renesse and Scheutzow (2010) states a stochastic Gronwall inequality in the case of continuous $M, Z, H$ which is less general than part (b) in Theorem 2.1. In addition, the proof of von Renesse and Scheutzow (2010, Lemma 5.4) contains a gap since the processes $X_{i}$ defined there can be negative outside of $\Omega_{i}$.

Counterexample 2.4. Under the assumptions of Theorem 2.1, for $p, \alpha \in(0,1)$, the inequality

$$
\mathbb{E}\left[\left(Z^{*}(T)\right)^{p} \mid \mathcal{F}_{0}\right] \leq c_{1, p, \alpha}\left(\mathbb{E}\left[(H(T))^{\alpha} \mid \mathcal{F}_{0}\right]\right)^{p / \alpha} \exp \left\{c_{2, p, \alpha} A(T)\right\}
$$

is generally not true with finite constants $c_{1, p, \alpha}$ and $c_{2, p, \alpha}$ for càdlàg martingales without assuming predictability of $H$. To see this, let $q \in(0,1)$ and let $S_{q, \alpha}$ be a random variable such that

$$
S_{q, \alpha}= \begin{cases}(1-q)^{1-\frac{1}{\alpha}} q^{-1}, & \text { with probability } q ; \\ -(1-q)^{-\frac{1}{\alpha}}, & \text { with probability } 1-q .\end{cases}
$$

Consider $M_{q, \alpha}(t):=\mathbf{1}_{[1, \infty)}(t) S_{q, \alpha}, H_{q, \alpha}(t):=\mathbf{1}_{[1, \infty)}(t)\left(S_{q, \alpha}\right)_{-}\left(\right.$with $x_{-}:=(-x) \vee$ $0, x \in \mathbb{R})$ and $Z_{q, \alpha}(t):=M_{q, \alpha}(t)+H_{q, \alpha}(t)$. Then there is no constant $c_{p, \alpha}$ depending only on $p, \alpha \in(0,1)$ such that the inequality

$$
\mathbb{E}\left[\left(Z_{q, \alpha}^{*}(1)\right)^{p}\right] \leq c_{p, \alpha}\left(\mathbb{E}\left[\left(H_{q, \alpha}(1)\right)^{\alpha}\right]\right)^{p / \alpha}
$$

holds for all $q \in(0,1)$ since

$$
\mathbb{E}\left[\left(Z_{q, \alpha}^{*}(1)\right)^{p}\right]=\mathbb{E}\left[\left(S_{q, \alpha}\right)_{+}^{p}\right]=(1-q)^{p\left(1-\frac{1}{\alpha}\right)} q^{1-p} \rightarrow \infty, \quad \text { as } q \rightarrow 1,
$$

while, on the other hand,

$$
\mathbb{E}\left[\left(H_{q, \alpha}(1)\right)^{\alpha}\right]=\mathbb{E}\left(\left(S_{q, \alpha}\right)_{-}^{\alpha}\right)=1 .
$$




\section{Well-posedness of Path-dependent SDEs}

First, we recall the definition of an orthogonal martingale-valued measure according to El Karoui and Méléard (1990); Walsh (1986). Let $(U, \mathcal{U})$ be a Lusin space, i.e. a measurable space homeomorphic to a Borel subset of $\mathbb{R}$. Consider an increasing sequence $U_{n}, n \in \mathbb{N}$ in $\mathcal{U}$ such that $U=\cup_{n \in \mathbb{N}} U_{n}$ and define $\mathcal{U}_{n}:=\left.\mathcal{U}\right|_{U_{n}}$ and $\mathcal{A}:=\cup_{n \in \mathbb{N}} \mathcal{U}_{n}$. A martingale measure is a set function $\tilde{M}: \mathbb{R}^{+} \times \mathcal{A} \times \Omega \rightarrow \mathbb{R}$ which satisfies the following (c.f. Applebaum, 2006; El Karoui and Méléard, 1990; Walsh, 1986):

(a) $\tilde{M}(0, A)=\tilde{M}(t, \emptyset)=0$ (a.s.), for all $A \in \mathcal{A}, t \geq 0$;

(b) $\tilde{M}(t, A \cup B)=\tilde{M}(t, A)+\tilde{M}(t, B)$ (a.s.), for all $t \geq 0$ and all disjoint $A, B \in \mathcal{A}$;

(c) For each non-increasing sequence $\left(A_{i}\right)$ of $\mathcal{U}_{n}$ converging to $\emptyset$, and for each $t \geq 0, \mathbb{E}\left[\left|\tilde{M}\left(t, A_{i}\right)\right|^{2}\right]$ tends to zero;

(d) $\sup \left\{\mathbb{E}|\tilde{M}(t, A)|^{2}, A \in \mathcal{U}_{n}\right\}<\infty$ for all $n \in \mathbb{N}$ and $t \geq 0$;

(e) $(\tilde{M}(t, A))_{t \geq 0}$ is a càdlàg martingale for all $A \in \mathcal{A}$.

Note that $\tilde{M}$ is countably additive on $\mathcal{U}_{n}$ as an $L^{2}$-valued set function. In Walsh's terminology (Walsh, 1986), $\tilde{M}$ is called " $\sigma$-finite $L^{2}$-valued martingale measure".

A martingale measure $\tilde{M}$ is called orthogonal if for all $A, B \in \mathcal{A}$ with $A \cap B=\emptyset$, $\left(\tilde{M}_{t}(A) \cdot \tilde{M}_{t}(B)\right)_{t \geq 0}$ is a martingale. Note that in this case property (d) holds automatically.

Throughout the paper, $\nu: \mathbb{R}^{+} \times \mathcal{U} \rightarrow \mathbb{R} \cup\{+\infty\}$ denotes a deterministic function such that for each $t \geq 0, \nu(t, \cdot)$ is a $\sigma$-finite measure and the map $t \mapsto \nu(t, A)$ is measurable and locally integrable for each $A \in \mathcal{A}$. We assume that $\tilde{M}$ is an orthogonal martingale measure with intensity $\left(\nu_{t}\right)_{t \geq 0}$, i.e. $\langle\tilde{M} \cdot(A), \tilde{M} .(B)\rangle_{t}=$ $\int_{0}^{t} \nu_{r}(A \cap B) \mathrm{d} r$, which means $\left(\tilde{M}(t, A) \tilde{M}(t, B)-\int_{0}^{t} \nu_{r}(A \cap B) \mathrm{d} r\right)_{t \geq 0}$ is a martingale for all $A, B \in \mathcal{A}$.

The stochastic integral with respect to $\tilde{M}$ can be constructed in the same way as the construction of Itô's integral (see Walsh, 1986). In particular, the stochastic integral $h \cdot \tilde{M}$ is defined for functions $h$ in

$$
\begin{aligned}
L_{\nu}^{2}:=\left\{h:\left(\mathbb{R}^{+} \times \Omega \times U, \mathcal{P} \otimes \mathcal{U}\right) \rightarrow\right. & \left(\mathbb{R}^{d}, \mathcal{B}\left(\mathbb{R}^{d}\right)\right) ; \\
& \left.\mathbb{E} \int_{0}^{T} \int_{U}|h(s, \omega, \xi)|^{2} \nu_{s}(\mathrm{~d} \xi) \mathrm{d} s<\infty, \forall T>0\right\},
\end{aligned}
$$

where $\mathcal{P}$ denotes the predictable $\sigma$-field on $\mathbb{R}^{+} \times \Omega$. Further, $h \cdot \tilde{M}$ is itself an orthogonal martingale measure and we have

$$
\langle h \cdot \tilde{M} \cdot(A), h \cdot \tilde{M} \cdot(B)\rangle_{t}=\int_{0}^{t}|h(s, \omega, \xi)|^{2} \nu_{s}(A \cap B) \mathrm{d} s .
$$

Applying the usual localization procedure, the class of admissible integrands can be further extended to the class of measurable functions $h:\left(\mathbb{R}^{+} \times \Omega \times U, \mathcal{P} \otimes \mathcal{U}\right) \rightarrow$ $\left(\mathbb{R}^{d}, \mathcal{B}\left(\mathbb{R}^{d}\right)\right)$ for which $\int_{0}^{T} \int_{U}|h(s, \omega, \xi)|^{2} \nu_{s}(\mathrm{~d} \xi) \mathrm{d} s<\infty, \forall T>0$, almost surely. In this case, (3.1) still holds. 
Now we are ready to provide a general existence and uniqueness result on strong solutions of functional stochastic differential equations with monotone coefficients driven by (orthogonal) martingale noise as above.

Consider the following path-dependent stochastic differential equation

$$
\left\{\begin{array}{l}
\mathrm{d} X_{t}=f(t, \omega, X) \mathrm{d} t+\int_{U} g(t, \omega, X, \xi) \tilde{M}(\mathrm{~d} t, \mathrm{~d} \xi), \\
X_{t}=z_{t}, \quad t \in[-\tau, 0]
\end{array}\right.
$$

where $\tau>0$ and the random initial condition $z$ belongs to Càdlàg $\left([-\tau, 0] ; \mathbb{R}^{d}\right)$ and is $\mathcal{F}_{0}$ measurable. All spaces of càdlàg functions are endowed with the supremum norm. The coefficient

$$
\begin{aligned}
& f:\left([0, \infty) \times \Omega \times \text { Càdlàg }\left([-\tau, \infty) ; \mathbb{R}^{d}\right), \mathcal{B F} \otimes \mathcal{B}\left(\text { Càdlàg }\left([-\tau, \infty) ; \mathbb{R}^{d}\right)\right)\right) \\
& \quad \rightarrow\left(\mathbb{R}^{d}, \mathcal{B}\left(\mathbb{R}^{d}\right)\right)
\end{aligned}
$$

is progressively measurable and

$$
\begin{aligned}
g: & \left([0, \infty) \times \Omega \times \text { Càdlàg }\left([-\tau, \infty) ; \mathbb{R}^{d}\right) \times U, \mathcal{P} \otimes \mathcal{B}\left(\text { Càdlàg }\left([-\tau, \infty) ; \mathbb{R}^{d}\right)\right) \otimes \mathcal{U}\right) \\
& \rightarrow\left(\mathbb{R}^{d}, \mathcal{B}\left(\mathbb{R}^{d}\right)\right)
\end{aligned}
$$

is predictable. Here $\mathcal{B F}$ is the $\sigma$-field of progressively measurable sets on $[0, \infty) \times \Omega$. For every $t \in[0, \infty)$ and $\omega \in \Omega, f(t, \omega, x)$ depends only on the path of $x$ on the interval $[-\tau, t]$ and for every $t, \omega, \xi, g(t, \omega, x, \xi)$ depends only on the path of $x$ on the interval $[-\tau, t)$.

The following monotonicity and growth conditions are assumed:

Hypothesis 3.1. There exist non-negative functions $t \mapsto K(t)$ and, for all $R>0$, $L_{R}(t)$ and $\tilde{K}_{R}(t)$ in $L_{\mathrm{loc}}^{1}([0, \infty), \mathrm{d} t)$ such that for all $x, y \in C \grave{a} d l \grave{g} g\left([-\tau, \infty), \mathbb{R}^{d}\right)$ and all $t \geq 0$,

(C1) for $\sup _{s \in[-\tau, t]}|x(s)|, \sup _{s \in[-\tau, t]}|y(s)| \leq R$,

$2\left\langle x\left(t^{-}\right)-y\left(t^{-}\right), f(t, \omega, x)-f(t, \omega, y)\right\rangle+\int_{U}|g(t, \omega, x, \xi)-g(t, \omega, y, \xi)|^{2} \nu_{t}(\mathrm{~d} \xi)$

$$
\leq L_{R}(t) \sup _{s \in[-\tau, t]}|x(s)-y(s)|^{2}
$$

(C2) $2\left\langle x\left(t^{-}\right), f(t, \omega, x)\right\rangle+\int_{U}|g(t, \omega, x, \xi)|^{2} \nu_{t}(\mathrm{~d} \xi) \leq K(t)\left(1+\sup _{s \in[-\tau, t]}|x(s)|^{2}\right)$;

(C3) $x \mapsto f(t, \omega, x)$ as a function from Càdlàg $\left([-\tau, \infty) ; \mathbb{R}^{d}\right)$ to $\mathbb{R}^{d}$ is continuous;

(C4) for $\sup _{s \in[-\tau, t]}|x(s)| \leq R$,

$$
|f(t, \omega, x)|+\int_{U}|g(t, \omega, x, \xi)|^{2} \nu_{t}(\mathrm{~d} \xi) \leq \tilde{K}_{R}(t)
$$

(C5) $\mathbb{E} \sup _{s \in[-\tau, 0]}|z(s)|^{2}<\infty$.

Remark 3.2. Conditions (C1) and (C3) imply that $x \mapsto g(t, \omega, x, \cdot)$ as a function from Càdlàg $\left([-\tau, \infty) ; \mathbb{R}^{d}\right)$ to $L^{2}\left(U, \mathcal{U}, \nu_{t}\right)$ is continuous.

We are going to prove existence and uniqueness of a strong solution using the Euler method. To this end let us introduce for $n \in \mathbb{N}$ and $k \in \mathbb{N}_{0}$ the Euler 
approximation

$$
\begin{aligned}
X_{t}^{(n)}= & X_{\frac{k}{n}}^{(n)}+\int_{\frac{k}{n}}^{t} f\left(s, \omega, X_{\cdot \wedge \frac{k}{n}}^{(n)}\right) \mathrm{d} s \\
& \left.\left.+\int_{\left(\frac{k}{n}, t\right] \times U} g\left(s, \omega, X_{\cdot \wedge \frac{k}{n}}^{(n)}, \xi\right) \tilde{M}(\mathrm{~d} s, \mathrm{~d} \xi), \quad t \in\right] \frac{k}{n}, \frac{k+1}{n}\right],
\end{aligned}
$$

to the solution of (3.2). Let $\kappa(n, t):=\frac{k}{n}$ for $\left.\left.t \in\right] \frac{k}{n}, \frac{k+1}{n}\right], k \geq 0$ and $\kappa(n, t):=t$ for $t \in[-\tau, 0]$. The process $X^{(n)}$ can be constructed inductively as follows: $X_{t}^{(n)}:=z_{t}$ for $t \in[-\tau, 0]$, and given $X_{t}^{(n)}$ is defined for $t \leq \frac{k}{n}$ we can extend $X_{t}^{(n)}$ for $t \in$ ]$\left.\frac{k}{n}, \frac{k+1}{n}\right]$ using (3.3). Note that $X^{(n)}, t \geq-\tau$ is càdlàg, adapted, and that the stochastic integrals are well-defined.

Theorem 3.3. Under Hypothesis 3.1, equation (3.2) has a unique strong solution $X$, and $X^{(n)}$ converges to $X$ locally uniformly in probability, i.e. for all $T>0$,

$$
\lim _{n \rightarrow \infty} \mathbb{P}\left\{\sup _{t \in[0, T]}\left|X_{t}^{(n)}-X_{t}\right|>\varepsilon\right\}=0 \quad \forall \varepsilon>0 .
$$

Proof: Let us define the remainder

$$
p_{t}^{(n)}=X_{\kappa(n, t)}^{(n)}-X_{t}^{(n)}, \quad t \in[-\tau, \infty) .
$$

Then $p^{(n)}$ is adapted and $p^{(n)}\left((k / n)^{+}\right)=0$ for every $k \in \mathbb{N}_{0}$. Further,

$$
X_{t}^{(n)}=z_{0}+\int_{0}^{t} f\left(s, \omega, X_{\cdot \wedge \kappa(n, s)}^{(n)}\right) \mathrm{d} s+\int_{0}^{t} \int_{U} g\left(s, \omega, X_{\cdot \wedge \kappa(n, s)}^{(n)}, \xi\right) \tilde{M}(\mathrm{~d} s, \mathrm{~d} \xi) .
$$

Fix $T>0$ and define the stopping times

$$
\tau_{R}^{(n)}:=\left(\inf \left\{t \geq 0:\left|X_{t}^{(n)}\right|>\frac{R}{3}\right\} \wedge T\right) \mathbf{1}_{\left\{R>3 \sup _{s \in[-\tau, 0]}|z(s)|\right\}}
$$

for given $R>0$. Then

$$
\left|p_{t}^{(n)}\right| \leq \frac{2 R}{3},\left|X_{t}^{(n)}\right| \leq \frac{R}{3}, \quad t \in\left(0, \tau_{R}^{(n)}\right) .
$$

For $R>3 \sup _{s \in[-\tau, 0]}|z(s)|$ the above inequalities extend to all $t \in\left[-\tau, \tau_{R}^{(n)}\right)$ and $\tau_{R}^{(n)}>0$ due to the right continuity of $X_{t}^{(n)}$.

We will prove the following properties which complete the proof of existence on $[0, T]$, and hence on $[0, \infty)$, since $T$ was arbitrary.

(i) For every $t \geq 0, \mathbf{1}_{\left(0, \tau_{R}^{(n)}\right)}(t) \sup _{u \in(\kappa(n, t), t]}\left|p_{u}^{(n)}\right| \rightarrow 0$ in probability as $n \rightarrow$

(ii) $\mathbb{E} \sup _{u \in[0, T]}\left|X_{u \wedge \tau_{R}^{(n)}}^{(n)}\right|^{2 p} \leq C(T, R, n, p)$, for some $C(T, R, n, p)$ satisfying

$$
\lim _{n \rightarrow \infty} C(T, R, n, p)=\tilde{C}(T, p) \text { for all } p \in(0,1), R>0 .
$$

(iii) $\lim _{R \rightarrow \infty} \lim \sup _{n \rightarrow \infty} \mathbb{P}\left\{\tau_{R}^{(n)}<T\right\}=0$.

(iv) $\forall \varepsilon>0, \lim _{n, m \rightarrow \infty} \mathbb{P}\left\{\sup _{t \in[0, T]}\left|X_{t}^{(n)}-X_{t}^{(m)}\right|>\varepsilon\right\}=0$. 
(v) $\exists X: \forall \varepsilon>0, \lim _{n \rightarrow \infty} \mathbb{P}\left\{\sup _{t \in[0, T]}\left|X_{t}^{(n)}-X_{t}\right|>\varepsilon\right\}=0$ and $X$ is a strong solution of equation $(3.2)$ on $[0, T]$.

Proof of (i): Fix $t>0$ and $\varepsilon>0$. Using (3.3) and Hypothesis 3.1 (C4), we have

$$
\begin{aligned}
& \mathbb{P}\left\{\sup _{u \in(\kappa(n, t), t]}\left|p_{u}^{(n)}\right| \geq \varepsilon, \tau_{R}^{(n)}>t\right\} \\
& \leq \mathbb{P}\left\{\int_{\kappa(n, t)}^{t}\left|f\left(s, \omega, X_{\cdot \wedge \kappa(n, s)}^{(n)}\right)\right| \mathrm{d} s \geq \varepsilon / 2, \tau_{R}^{(n)}>t\right\} \\
& +\mathbb{P}\left\{\left|\sup _{u \in(\kappa(n, t), t]} \int_{\kappa(n, t)}^{u} \int_{U} \mathbf{1}_{\left\{s \leq \tau_{R}^{(n)}\right\}} g\left(s, \omega, X_{\cdot \wedge \kappa(n, s)}^{(n)}, \xi\right) \tilde{M}(\mathrm{~d} s, \mathrm{~d} \xi)\right| \geq \varepsilon / 2, \tau_{R}^{(n)}>t\right\} \\
& \leq \mathbb{P}\left\{\int_{\kappa(n, t)}^{t} \tilde{K}_{R}(s) \mathrm{d} s \geq \varepsilon / 2\right\} \\
& +\frac{4}{\varepsilon^{2}} \mathbb{E}\left(\sup _{u \in(\kappa(n, t), t]}\left|\int_{\kappa(n, t)}^{u} \int_{U} \mathbf{1}_{\left\{s \leq \tau_{R}^{(n)}\right\}} g\left(s, \omega, X_{\cdot \wedge \kappa(n, s)}^{(n)}, \xi\right) \tilde{M}(\mathrm{~d} s, \mathrm{~d} \xi)\right|^{2}\right)
\end{aligned}
$$

Using Burkholder-Davis-Gundy's inequality, we continue as follows

$$
\begin{aligned}
& \leq \frac{2}{\varepsilon} \int_{\kappa(n, t)}^{t} \tilde{K}_{R}(s) \mathrm{d} s+\frac{C}{\varepsilon^{2}} \mathbb{E}\left[\int_{\kappa(n, t)} \int_{U} \mathbf{1}_{\left\{s \leq \tau_{R}^{(n)}\right\}} g\left(s, \omega, X_{\cdot \wedge \kappa(n, s)}^{(n)}, \xi\right) \tilde{M}(\mathrm{~d} s, \mathrm{~d} \xi)\right]_{t} \\
& \leq \frac{2}{\varepsilon} \int_{\kappa(n, t)}^{t} \tilde{K}_{R}(s) \mathrm{d} s+\frac{C}{\varepsilon^{2}} \mathbb{E} \int_{\kappa(n, t)}^{t} \int_{U} \mathbf{1}_{\left\{s \leq \tau_{R}^{(n)}\right\}}\left|g\left(s, \omega, X_{\cdot \wedge \kappa(n, s)}^{(n)}, \xi\right)\right|^{2} \nu_{s}(\mathrm{~d} \xi) \mathrm{d} s \\
& \leq \frac{2}{\varepsilon} \int_{\kappa(n, t)}^{t} \tilde{K}_{R}(s) \mathrm{d} s+\frac{C}{\varepsilon^{2}} \mathbb{E}\left(\int_{\kappa(n, t)}^{t} \tilde{K}_{R}(s) \mathrm{d} s\right)=\left(\frac{2}{\varepsilon}+\frac{C}{\varepsilon^{2}}\right) \int_{\kappa(n, t)}^{t} \tilde{K}_{R}(s) \mathrm{d} s,
\end{aligned}
$$

so

$$
\limsup _{n \rightarrow \infty} \mathbb{P}\left\{\sup _{u \in(\kappa(n, t), t]}\left|p_{u}^{(n)}\right| \geq \varepsilon, \tau_{R}^{(n)}>t\right\}=0
$$

which implies (i) since $\varepsilon>0$ was arbitrary.

Proof of (ii): Using Itô's formula, we obtain

$$
\begin{aligned}
\left|X_{t}^{(n)}\right|^{2}= & \left|z_{0}\right|^{2}+\int_{0}^{t} 2\left\langle X_{s-}^{(n)}, f\left(s, \omega, X_{\cdot \wedge \kappa(n, s)}^{(n)}\right)\right\rangle \mathrm{d} s \\
& +\int_{0}^{t} \int_{U}\left|g\left(s, \omega, X_{\cdot \wedge \kappa(n, s)}^{(n)}, \xi\right)\right|^{2} \nu_{s}(\mathrm{~d} \xi) \mathrm{d} s+M_{t}^{(n)} .
\end{aligned}
$$

where

$$
\begin{aligned}
M_{t}^{(n)}:= & \int_{0}^{t} \int_{U} 2\left\langle X_{s-}^{(n)}, g\left(s, \omega, X_{\cdot \wedge \kappa(n, s)}^{(n)}, \xi\right)\right\rangle \tilde{M}(\mathrm{~d} s, \mathrm{~d} \xi) \\
& +\left[\int_{0} \int_{U} g\left(s, \omega, X_{\cdot \wedge \kappa(n, s)}^{(n)}, \xi\right) \tilde{M}(\mathrm{~d} s, \mathrm{~d} \xi)\right]_{t} \\
& -\int_{0}^{t} \int_{U}\left|g\left(s, \omega, X_{\cdot \wedge \kappa(n, s)}^{(n)}, \xi\right)\right|^{2} \nu_{s}(\mathrm{~d} \xi) \mathrm{d} s
\end{aligned}
$$


and $\left(M_{t \wedge \tau_{R}^{(n)}}^{(n)}\right)_{t \geq 0}$ is a local martingale. Using (C2) and (C4), we have

$$
\begin{aligned}
\left|X_{t \wedge \tau_{R}^{(n)}}^{(n)}\right|^{2} \leq & \left|z_{0}\right|^{2}+\int_{0}^{t \wedge \tau_{R}^{(n)}} 2\left\langle X_{s^{-}}^{(n)}-X_{\kappa(n, s)}^{(n)}, f\left(s, \omega, X_{\cdot \wedge \kappa(n, s)}^{(n)}\right)\right\rangle \mathrm{d} s \\
& +\int_{0}^{t \wedge \tau_{R}^{(n)}} K(s)\left(1+\sup _{u \in[-\tau, s]}\left|X_{u}^{(n)}\right|^{2}\right) \mathrm{d} s+M_{t \wedge \tau_{R}^{(n)}}^{(n)} \\
\leq & \left|z_{0}\right|^{2}+2 \int_{0}^{t} \mathbf{1}_{\left\{s \in\left(0, \tau_{R}^{(n)}\right]\right\}} \tilde{K}_{R}(s)\left|p_{s}^{(n)}\right| \mathrm{d} s \\
& +\int_{0}^{t} K(s)\left[1+\sup _{u \in[-\tau, 0]}\left|z_{u}\right|^{2}+\sup _{u \in[0, s]}\left|X_{u \wedge \tau_{R}^{(n)}}^{(n)}\right|^{2}\right] \mathrm{d} s+M_{t \wedge \tau_{R}^{(n)}}^{(n)} \\
= & \int_{0}^{t} K(s) \sup _{u \in[0, s]}\left|X_{u \wedge \tau_{R}^{(n)}}^{(n)}\right|^{2} \mathrm{~d} s+H_{t}^{n, R}+M_{t \wedge \tau_{R}^{(n)}}^{(n)} .
\end{aligned}
$$

where

$$
H_{t}^{n, R}:=\left|z_{0}\right|^{2}+\int_{0}^{t}\left[K(s)\left(1+\sup _{u \in[-\tau, 0]}\left|z_{u}\right|^{2}\right)+2 \cdot \mathbf{1}_{\left\{s \in\left(0, \tau_{R}^{(n)}\right]\right\}} \tilde{K}_{R}(s)\left|p_{s^{-}}^{(n)}\right|\right] \mathrm{d} s .
$$

Using Theorem 2.1, we get for $p \in(0,1)$ that

$$
\mathbb{E}\left[\sup _{u \in[0, T]}\left|X_{u \wedge \tau_{R}^{(n)}}^{(n)}\right|^{2 p}\right] \leq C(T, p)\left(\mathbb{E} H_{T}^{n, R}\right)^{p}=: C(T, R, n, p)
$$

where, by (i) and the dominated convergence theorem,

$$
\lim _{n \rightarrow \infty} \mathbb{E} H_{T}^{n, R}=\mathbb{E}\left[\left|z_{0}\right|^{2}+\int_{0}^{T} K(s)\left(1+\sup _{u \in[-\tau, 0]}\left|z_{u}\right|^{2}\right) \mathrm{d} s\right]
$$

Hence $\lim _{n \rightarrow \infty} C(T, R, n, p)=: \tilde{C}(T, p)$ exists and is independent of $R>0$ and hence (ii) holds.

Proof of (iii): We have, for $p \in(0,1)$,

$$
\begin{aligned}
& \limsup _{R \rightarrow \infty} \limsup _{n \rightarrow \infty} \mathbb{P}\left\{\sup _{t \in\left[0, \tau_{R}^{(n)}\right]}\left|X_{t}^{(n)}\right| \geq \frac{R}{4} ; \tau_{R}^{(n)}<T\right\} \\
& \quad \leq \limsup _{R \rightarrow \infty} \limsup _{n \rightarrow \infty} \mathbb{P}\left\{\sup _{t \in\left[0, T \wedge \tau_{R}^{(n)}\right]}\left|X_{t}^{(n)}\right| \geq \frac{R}{4}\right\} \\
& \quad \leq \limsup _{R \rightarrow \infty}\left(\frac{4}{R}\right)^{2 p} \underset{n \rightarrow \infty}{\limsup C(T, R, n, p) \leq \limsup _{R \rightarrow \infty}\left(\frac{4}{R}\right)^{2 p} \tilde{C}(T, p)=0 .}
\end{aligned}
$$

It follows that

$$
\limsup _{R \rightarrow \infty} \limsup _{n \rightarrow \infty} \mathbb{P}\left\{\tau_{R}^{(n)}<T\right\} \leq \limsup _{R \rightarrow \infty} \limsup _{n \rightarrow \infty} \mathbb{P}\left\{\sup _{t \in\left[0, \tau_{R}^{(n)}\right]}\left|X_{t}^{(n)}\right| \geq \frac{R}{4} ; \tau_{R}^{(n)}<T\right\}=0
$$

which completes the proof of (iii). 
Proof of (iv): Let $\tau_{R}^{n, m}:=\tau_{R}^{(n)} \wedge \tau_{R}^{(m)}$. Using Itô's formula, we have

$$
\begin{aligned}
& \left|X_{t}^{(n)}-X_{t}^{(m)}\right|^{2} \\
& \quad=M_{t}^{n, m}+\int_{0}^{t} 2\left\langle X_{s^{-}}^{(n)}-X_{s^{-}}^{(m)}, f\left(s, \omega, X_{\cdot \wedge \kappa(n, s)}^{(n)}\right)-f\left(s, \omega, X_{\cdot \wedge \kappa(m, s)}^{(m)}\right)\right\rangle \mathrm{d} s \\
& \quad+\int_{0}^{t} \int_{U}\left|g\left(s, \omega, X_{\cdot \wedge \kappa(n, s)}^{(n)}, \xi\right)-g\left(s, \omega, X_{\cdot \wedge \kappa(m, s)}^{(m)}, \xi\right)\right|^{2} \nu_{s}(\mathrm{~d} \xi) \mathrm{d} s
\end{aligned}
$$

where $\left(M_{t \wedge \tau_{R}^{n, m}}^{n, m}\right)_{t \geq 0}$ is a local martingale starting from zero. Hypothesis (C1) implies

$$
\begin{aligned}
& \left|X_{t \wedge \tau_{R}^{n, m}}^{(n)}-X_{t \wedge \tau_{R}^{n, m}}^{(m)}\right|^{2} \\
& \leq M_{t \wedge \tau_{R}^{n, m}}^{n, m}+\int_{0}^{t \wedge \tau_{R}^{n, m}} 2\left\langle X_{s^{-}}^{(n)}-X_{s^{-}}^{(m)}-X_{\kappa(n, s)}^{(n)}+X_{\kappa(m, s)}^{(m)},\right. \\
& \left.f\left(s, \omega, X_{\cdot \wedge \kappa(n, s)}^{(n)}\right)-f\left(s, \omega, X_{\cdot \wedge \kappa(m, s)}^{(m)}\right)\right\rangle \mathrm{d} s \\
& +\int_{0}^{t \wedge \tau_{R}^{n, m}} L_{R}(s) \sup _{u \in[-\tau, s]}\left|X_{u}^{(n)}+\mathbf{1}_{(\kappa(n, s), s]}(u) p_{u}^{(n)}-X_{u}^{(m)}-\mathbf{1}_{(\kappa(m, s), s]} p_{u}^{(m)}\right|^{2} \mathrm{~d} s \\
& \leq M_{t \wedge \tau_{R}^{n, m}}^{n, m}+\int_{0}^{t} \mathbf{1}_{\left(0, \tau_{R}^{n, m}\right)}(s)\left\{4 \tilde{K}_{R}(s)\left|p_{s^{-}}^{(n)}+p_{s^{-}}^{(m)}\right|\right. \\
& \left.+3 L_{R}(s) R\left(\sup _{u \in(\kappa(n, s), s]}\left|p_{u}^{(n)}\right|+\sup _{u \in(\kappa(m, s), s]}\left|p_{u}^{(m)}\right|\right)\right\} \mathrm{d} s \\
& +\int_{0}^{t} L_{R}(s) \sup _{u \in[0, s]}\left|X_{u \wedge \tau_{R}^{n, m}}^{(n)}-X_{u \wedge \tau_{R}^{n, m}}^{(m)}\right|^{2} \mathrm{~d} s \\
& \leq M_{t \wedge \tau_{R}^{n, m}}^{n, m}+\int_{0}^{t} L_{R}(s) \sup _{u \in[0, s]}\left|X_{u \wedge \tau_{R}^{(n)}}^{(n, m}-X_{u \wedge \tau_{R}^{n, m}}^{(m)}\right|^{2} \mathrm{~d} s+H_{t}^{n, m, R},
\end{aligned}
$$

where

$$
\begin{aligned}
H_{t}^{n, m, R}:=\int_{0}^{t} \mathbf{1}_{\left(0, \tau_{R}^{n, m}\right)}(s) & \left\{4 \tilde{K}_{R}(s)\left|p_{s^{-}}^{(n)}+p_{s^{-}}^{(m)}\right|\right. \\
& \left.+3 L_{R}(s) R\left(\sup _{u \in(\kappa(n, s), s]}\left|p_{u}^{(n)}\right|+\sup _{u \in(\kappa(m, s), s]}\left|p_{u}^{(m)}\right|\right)\right\} \mathrm{d} s .
\end{aligned}
$$

Using Theorem 2.1, we have for $p \in(0,1)$ that

$$
\mathbb{E}\left[\sup _{t \in[0, T]}\left|X_{t \wedge \tau_{R}^{n, m}}^{(n)}-X_{t \wedge \tau_{R}^{(m)}}^{(m)}\right|^{2 p}\right] \leq C(T, R, p)\left(\mathbb{E} H_{T}^{n, m, R}\right)^{p} .
$$

Hence for $a>0$,

$$
\begin{aligned}
\mathbb{P}\left\{\sup _{t \in[0, T]}\left|X_{t}^{(n)}-X_{t}^{(m)}\right| \geq a\right\} \\
\quad \leq \mathbb{P}\left\{T>\tau_{R}^{(n)}\right\}+\mathbb{P}\left\{T>\tau_{R}^{(m)}\right\}+\mathbb{P}\left\{\sup _{t \in\left[0, \tau_{R}^{n, m}\right]}\left|X_{t}^{(n)}-X_{t}^{(m)}\right| \geq a\right\} \\
\quad \leq \mathbb{P}\left\{T>\tau_{R}^{(n)}\right\}+\mathbb{P}\left\{T>\tau_{R}^{(m)}\right\}+\frac{1}{a^{2 p}} \mathbb{E}\left[\sup _{t \in[0, T]}\left|X_{t \wedge \tau_{R}^{n, m}}^{(n)}-X_{t \wedge \tau_{R}^{n, m}}^{(m)}\right|^{2 p}\right] \\
\leq \mathbb{P}\left\{T>\tau_{R}^{(n)}\right\}+\mathbb{P}\left\{T>\tau_{R}^{(m)}\right\}+a^{-2 p} C(T, R, p)\left(\mathbb{E} H_{T}^{n, m, R}\right)^{p} .
\end{aligned}
$$


(i) and dominated convergence now imply that

$$
\limsup _{n, m \rightarrow \infty} \mathbb{E} H_{T}^{n, m, R}=0
$$

and using (iii), we get

$$
\begin{aligned}
& \limsup _{n, m \rightarrow \infty} \mathbb{P}\left\{\sup _{t \in[0, T]}\left|X_{t}^{(n)}-X_{t}^{(m)}\right| \geq a\right\} \\
& \leq \lim _{R \rightarrow \infty} \limsup _{n, m \rightarrow \infty}\left[\mathbb{P}\left\{T>\tau_{R}^{(n)}\right\}+\mathbb{P}\left\{T>\tau_{R}^{(m)}\right\}+a^{-2 p} C(T, R, p)\left(\mathbb{E} H_{T}^{n, m, R}\right)^{p}\right]=0,
\end{aligned}
$$

so (iv) is obtained.

Proof of $(\mathrm{v})$ : Since the space Càdlàg $\left([-\tau, T], \mathbb{R}^{d}\right)$ is complete, via the Borel-Cantelli lemma, (iv) yields that there exists an adapted càdlàg process $X$ such that

$$
\lim _{n \rightarrow \infty} \mathbb{P}\left\{\sup _{t \in[0, T]}\left|X_{t}^{(n)}-X_{t}\right| \geq \varepsilon\right\}=0 .
$$

We have to show that, for a subsequence of $n \in \mathbb{N}$, all terms of equation (3.4) converge almost surely to the corresponding terms of equation (3.2). We have

$$
\begin{aligned}
\limsup _{n \rightarrow \infty} & \int_{0}^{T} \mathbb{P}\left\{\sup _{u \in[0, t]}\left|X_{u \wedge \kappa(n, t)}^{(n)}-X_{u}\right| \geq \varepsilon\right\} \mathrm{d} t \\
\leq & \limsup _{n \rightarrow \infty} \int_{0}^{T} \mathbb{P}\left\{\sup _{u \in[0, T]}\left|X_{u}^{(n)}-X_{u}\right| \geq \varepsilon\right\} \mathrm{d} t+ \\
& +\limsup _{n \rightarrow \infty} \mathbb{E} \int_{0}^{T} \mathbf{1}_{\left\{\sup _{u \in(\kappa(n, t), t]}\left|X_{\kappa(n, t)}-X_{u}\right| \geq \varepsilon\right\}} \mathrm{d} t=0 .
\end{aligned}
$$

So we can find a subsequence, say $\left\{n_{l}\right\}_{l \in \mathbb{N}}$, such that as $l \rightarrow \infty$,

$$
\sup _{u \in[0, t]}\left|X_{u \wedge \kappa\left(n_{l}, t\right)}^{\left(n_{l}\right)}-X_{u}\right| \rightarrow 0 \quad \mathrm{~d} t \otimes \mathbb{P} \text {-a.e. }(t, \omega) \in[0, T] \times \Omega .
$$

Now let us define

$$
S(T):=\sup _{l \in \mathbb{N}} \sup _{t \in[0, T]} \sup _{u \in[0, t]}\left|X_{u \wedge \kappa\left(n_{l}, t\right)}^{\left(n_{l}\right)}\right|
$$

then

$$
S(T)<\infty \quad \mathbb{P} \text {-a.s. }
$$

Therefore, using (C3), (C4) and dominated convergence, we obtain that

$$
\lim _{l \rightarrow \infty} \int_{0}^{t} f\left(s, \omega, X_{\cdot \wedge \kappa\left(n_{l}, s\right)}^{\left(n_{l}\right)}\right) \mathrm{d} s=\int_{0}^{t} f(s, \omega, X) \mathrm{d} s \quad \mathbb{P} \text {-a.s. }
$$

Let $\tau(R):=\inf \{t \geq 0: S(t)>R\} \wedge T$. Fix $t \in[0, T]$. By (C4), Remark 3.2, and dominated convergence,

$$
\begin{aligned}
\lim _{l \rightarrow \infty} & \mathbb{E}\left|\int_{0}^{t \wedge \tau(R)} \int_{U}\left[g\left(s, \omega, X_{\cdot \wedge \kappa\left(n_{l}, s\right)}^{\left(n_{l}\right)}, \xi\right)-g(s, \omega, X, \xi)\right] \tilde{M}(\mathrm{~d} s, \mathrm{~d} \xi)\right|^{2} \\
& =\lim _{l \rightarrow \infty} \mathbb{E} \int_{0}^{t} \int_{U} \mathbf{1}_{\{s \leq \tau(R)\}}\left|g\left(s, \omega, X_{\cdot \wedge \kappa\left(n_{l}, s\right)}^{\left(n_{l}\right)}, \xi\right)-g(s, \omega, X, \xi)\right|^{2} \nu_{s}(\mathrm{~d} \xi) \mathrm{d} s=0
\end{aligned}
$$


so, for $t \in[0, T]$,

$$
\begin{aligned}
\mathbb{P}\left\{\mid \int_{0}^{t} \int_{U}\right. & {\left.\left[g\left(s, \omega, X_{\cdot \wedge \kappa\left(n_{l}, s\right)}^{\left(n_{\left.n_{l}\right)}\right.}, \xi\right)-g(s, \omega, X, \xi)\right] \tilde{M}(\mathrm{~d} s, \mathrm{~d} \xi) \mid>\varepsilon\right\} } \\
\leq & \mathbb{P}\left\{\left|\int_{0}^{t \wedge \tau(R)} \int_{U}\left[g\left(s, \omega, X_{\cdot \wedge \kappa\left(n_{l}, s\right)}^{\left(n_{l}\right)}, \xi\right)-g(s, \omega, X, \xi)\right] \tilde{M}(\mathrm{~d} s, \mathrm{~d} \xi)\right|>\varepsilon\right\} \\
+ & \mathbb{P}\{t>\tau(R)\} .
\end{aligned}
$$

Fix some sufficiently large $R$ such that the second term on the right hand side is less than $\delta>0$, then taking the limit $l \rightarrow \infty$ implies

$$
\lim _{l \rightarrow \infty} \mathbb{P}\left\{\left|\int_{0}^{t} \int_{U}\left[g\left(s, \omega, X_{\cdot \wedge \kappa\left(n_{l}, s\right)}^{\left(n_{l}\right)}, \xi\right)-g(s, \omega, X, \xi)\right] \tilde{M}(\mathrm{~d} s, \mathrm{~d} \xi)\right|>\varepsilon\right\} \leq \delta
$$

where $\delta>0$ is arbitrary. Therefore

$$
\int_{0}^{t} \int_{U} g\left(s, \omega, X_{\cdot \wedge \kappa\left(n_{l}, s\right)}^{\left(n_{l}\right)}, \xi\right) \tilde{M}(\mathrm{~d} s, \mathrm{~d} \xi) \rightarrow \int_{0}^{t} \int_{U} g(s, \omega, X, \xi) \tilde{M}(\mathrm{~d} s, \mathrm{~d} \xi)
$$

in probability and for some subsequence $n_{l_{k}}$ the above convergence is $\mathbb{P}$-a.s. Therefore $X$ is a solution of equation $(3.2)$ on $[0, T]$.

Uniqueness: Let $X$ and $Y$ be two solutions of equation (3.2) and define

$$
\tau(R):=\inf \left\{t \geq 0 ;\left|X_{t}\right|>R \text { or }\left|Y_{t}\right|>R\right\} .
$$

Then

$$
\begin{aligned}
\left|X_{t \wedge \tau(R)}-Y_{t \wedge \tau(R)}\right|^{2}= & \int_{0}^{t \wedge \tau(R)}\left\{2\left\langle X_{s^{-}}-Y_{s^{-}}, f(s, \omega, X)-f(s, \omega, Y)\right\rangle\right. \\
& \left.+\int_{U}|g(s, \omega, X, \xi)-g(s, \omega, Y, \xi)|^{2} \nu_{s}(\mathrm{~d} \xi)\right\} \mathrm{d} s+M_{t \wedge \tau(R)} \\
\leq & \int_{0}^{t} 1_{\{s \leq \tau(R)\}} L_{R}(s) \sup _{u \in[0, s]}\left|X_{u}-Y_{u}\right|^{2} \mathrm{~d} s+M_{t \wedge \tau(R)} \\
\leq & \int_{0}^{t} L_{R}(s) \sup _{u \in[0, s]}\left|X_{u \wedge \tau(R)}-Y_{u \wedge \tau(R)}\right|^{2} \mathrm{~d} s+M_{t \wedge \tau(R)}
\end{aligned}
$$

where $\left(M_{t \wedge \tau(R)}\right)_{t \geq 0}$ is a local martingale starting from zero. Using Theorem 2.1, for $p \in(0,1)$ we have

$$
\mathbb{E}\left[\sup _{s \in[0, T]}\left|X_{s \wedge \tau(R)}-Y_{s \wedge \tau(R)}\right|^{2 p}\right] \leq 0 .
$$

Therefore $X_{s \wedge \tau(R)}-Y_{s \wedge \tau(R)}=0$ a.s. and uniqueness is proved.

\section{An Example}

We provide a simple example satisfying the assumptions of Theorem 3.3 which does not seem to have been covered by previous results.

Example 4.1. Let $d=1, \tau=1$ and consider the equation

$$
\mathrm{d} X_{t}=\left[-\left|X_{t^{-}}\right|^{1 / 4}+\sup _{t-1 \leq s<t}\left|X_{s}\right|\right] \mathrm{d} t+\left[\left|X_{t^{-}}\right|^{3 / 4}+\sup _{t-1 \leq s<t}\left|X_{s}\right|\right] \mathrm{d} W_{t}+\mathrm{d} N_{t}
$$

where $W$ is standard Brownian motion and $N$ is compensated standard Poisson process.

It is straightforward to check that Hypothesis 3.1 is satisfied. Observe that the function $x \mapsto|x|^{3 / 4}$ does not satisfy a local Lipschitz condition in any neighborhood of 0 . To prove uniqueness of the solution, assume that $X$ and $Y$ are two solutions 
with initial condition 0 . Denoting their squared difference by $Z$, we arrive at an inequality of the form

$$
Z_{s} \leq K \int_{0}^{s} Z_{u}^{*} \mathrm{~d} u+M_{s}, s \geq 0,
$$

where the supremum in the integrand cannot be avoided. To apply the usual Gronwall inequality, we need to take the expectation of the supremum on both sides between 0 and $t$. Applying the Burkholder-Davis-Gundy inequality to estimate $\mathbb{E} M_{t}^{*}$ we thus arrive at an integral inequality for $\mathbb{E} Z_{t}^{*}$ to which we cannot apply the usual Gronwall lemma since, due to the lack of local Lipschitz continuity of the function $x \mapsto|x|^{3 / 4}$, the right hand side is too large. Without the Poisson term $N$, this equation is covered by the main result in von Renesse and Scheutzow (2010) by applying a stochastic Gronwall lemma for continuous processes. Due to the Poisson term $N$ this version of the stochastic Gronwall lemma cannot be applied to equation (4.1).

\section{References}

Applebaum, D. Martingale-valued measures, Ornstein-Uhlenbeck processes with jumps and operator self-decomposability in Hilbert space. In In memoriam PaulAndré Meyer: Séminaire de Probabilités XXXIX, volume 1874 of Lecture Notes in Math., pp. 171-196. Springer, Berlin (2006). MR2276896.

Chueshov, I. and Scheutzow, M. Invariance and monotonicity for stochastic delay differential equations. Discrete Contin. Dyn. Syst. Ser. B, 18 (6), 1533-1554 (2013). MR3038767.

Dellacherie, C. Capacités et processus stochastiques. Springer-Verlag, Berlin-New York (1972). MR0448504.

El Karoui, N. and Méléard, S. Martingale measures and stochastic calculus. Probab. Theory Related Fields, 84 (1), 83-101 (1990). MR1027822.

Fengying, W. and Ke, W. The existence and uniqueness of the solution for stochastic functional differential equations with infinite delay. J. Math. Anal. Appl., 331 (1), 516-531 (2007). MR2306021.

Kruse, R. and Scheutzow, M. A discrete stochastic Gronwall lemma. Math. Comput. Simulation, 143, 149-157 (2018). MR3698223.

Lenglart, E. Relation de domination entre deux processus. Ann. Inst. H. Poincaré Sect. B (N.S.), 13 (2), 171-179 (1977). MR0471069.

Mao, X. Stochastic differential equations and applications. Horwood Publishing Limited, Chichester, second edition (2008). ISBN 978-1-904275-34-3. MR2380366.

Mehri, S., Scheutzow, M., Stannat, W., and Zangeneh, B. Z. Propagation of chaos for stochastic spatially structured neuronal networks with delay driven by jump diffusions. Ann. Appl. Probab., 30 (1), 175-207 (2020). MR4068309.

Mohammed, S.-E. A. and Scheutzow, M. K. R. Lyapunov exponents of linear stochastic functional-differential equations. II. Examples and case studies. Ann. Probab., 25 (3), 1210-1240 (1997). MR1457617.

Mohammed, S.-E. A. and Scheutzow, M. K. R. The stable manifold theorem for non-linear stochastic systems with memory. I. Existence of the semiflow. J. Funct. Anal., 205 (2), 271-305 (2003). MR2017689. 
Ren, Y., Lu, S., and Xia, N. Remarks on the existence and uniqueness of the solutions to stochastic functional differential equations with infinite delay. $J$. Comput. Appl. Math., 220 (1-2), 364-372 (2008). MR2444176.

Ren, Y. and Shen, J. A note on the domination inequalities and their applications. Statist. Probab. Lett., 82 (6), 1160-1168 (2012). MR2915083.

Revuz, D. and Yor, M. Continuous martingales and Brownian motion, volume 293 of Grundlehren der Mathematischen Wissenschaften [Fundamental Principles of Mathematical Sciences]. Springer-Verlag, Berlin, third edition (1999). ISBN 3540-64325-7. MR1725357.

Scheutzow, M. A stochastic Gronwall lemma. Infin. Dimens. Anal. Quantum Probab. Relat. Top., 16 (2), 1350019, 4 (2013). MR3078830.

von Renesse, M.-K. and Scheutzow, M. Existence and uniqueness of solutions of stochastic functional differential equations. Random Oper. Stoch. Equ., 18 (3), 267-284 (2010). MR2718125.

Walsh, J. B. An introduction to stochastic partial differential equations. In École d'été de probabilités de Saint-Flour, XIV-1984, volume 1180 of Lecture Notes in Math., pp. 265-439. Springer, Berlin (1986). MR876085.

Zhang, X. and Zhao, G. Singular Brownian diffusion processes. Commun. Math. Stat., 6 (4), 533-581 (2018). MR3877717.

$\mathrm{Zhu}, \mathrm{Q}$. Razumikhin-type theorem for stochastic functional differential equations with Lévy noise and Markov switching. Internat. J. Control, 90 (8), 1703-1712 (2017). MR3658475. 